\title{
Exploring Duchampian and Darwinian ideas through interactive means
}

\author{
Dew Harrison \\ Associate Dean Postgraduate Studies and Research \\ University of Wolverhampton \\ School of Art \& Design \\ dew.harrison@wlv.ac.uk
}

\begin{abstract}
Within my practice-led research I continue to explore and further ideas from the fields of computermediated art and consciousness studies. This has recently developed as a merging of Art and Artificial Intelligence (Al), exampled through two projects currently underway, one Duchampian, the other Darwinian. These two projects share the idea that separate components, which are thematically unified through semantically associative media, can provide new holistic understandings of complex issues. As technologies have developed, interactivity has moved from screen and mouse to the more intuitive approach of sensor enabled physical activity and projection, and from hypermedia software to Al programming.
\end{abstract}

The Duchamp project: This focuses on his 'Large Glass'. By applying Al 'behaviours' to the objects in this piece, they move towards or away from each other according to their semantic relationships when in close proximity. These 'flocked' Duchampian objects more clearly show the 'families' of images, texts etc., and the oscillations that occur between them due to the pull of their similar semantic positions. The experiments with semantic media have now extended from the ideas of Duchamp to approach the highly creative 'big idea' of evolution through adaptation, in order to respond to Shrewsbury Museum Service's commission.

The Darwin Project: As part of the national Darwin celebrations underway, Shift-Life was commissioned for exhibit at Shift-Time - a festival of ideas in Shrewsbury, summer 2009. This interactive installation focuses on 'hands-on' possibilities for witnessing an evolutionary process in alternate life forms as they struggle to adapt to a volatile environment. Al behaviours were attached to a virtual world of fantasy animated objects featured as 2D 'jelly sweet' creatures and 'pick and mix sweet' plants. Their self-sustaining world is projected into a wooden box where visitors can cause physical upheavals in their virtual environment, by pouring liquids, hammering and turning lights on and off, to which the bug-like creatures respond instantly.

In moving from Duchamp to Darwin the approach has remained the same in that viewer engagement is paramount and the work is interactive where the participating visitor's actions and choices contribute to the delivery of the piece's content. Although there is a facade of entertainment, in that these artworks are quite playful and engaging, the intent remains of harnessing new technologies as creative systems to present and elucidate more complex ideas about art and life.

Hypermedia art systems. Duchamp. Darwin. Intuitive interfaces. Al behaviours

\section{INTRODUCTION}

In keeping with a conceptual practice I continue to question and analyse the idea of art. My work concerns the use of computer technology to augment our thinking and elucidate deeper understandings of issues and positions within the art field. The aptitude for the use of computers in Conceptual Art practice was initially voiced by Christine Tamblyn in an article for Art Journal, where she stated that computers were designed to augment mental processes as opposed to being visual or manual aids (Tamblyn, 1990). This understanding is in line with my own research and practice where I see both Conceptual Art and hypermedia dealing with the semantic association of ideas and thoughts in one interconnected narrative, or artwork. Hypermedia being an evolving conception to facilitate the augmentation of human mental activity by emulating organic memory systems. My interests lie in the extent to which computers are most efficiently engaged in 
contemporary art practice through a symbiotic relationship with Conceptualism and that this is most apparent where both computer-mediated and Conceptual Art have been influenced by the work and ideas of Marcel Duchamp. Duchamp being the instigator of the move to Conceptual practice in that he began the shift of values within art from visual aesthetics to idea.

My practice deals specifically with the semantic association or 'contextual linkage' of ideas and thoughts into one interconnected narrative or artwork in multimedia form, which mimics human thought and memory retrieval. Texts, images, animations and sounds are networked into one overarching 'concept'. The complete concept is then exhibited as a looped projected film or nonlinear screen work offering a contemporary understanding of a complex issue. Participation is paramount here and much of my work is interactive where the visitor's actions and choices contribute to the delivery of a piece's content. Although there is a façade of entertainment in that the pieces are quite playful and engaging, the intent is to use new technology to present and help elucidate more complex ideas about what art is. Within this practice I have been exploring the work and ideas of Marcel Duchamp and how new technologies are best suited to enable this re-articulation. This situates and compounds the value of text and wordplay, indexing and database, archiving and curation as both content and medium for a contemporary Conceptual practice.

\section{DUCHAMP}

Arguably, the most complex piece of art to date has been Duchamp's Large Glass entitled La Mariée mise à nu par ses célibataires, même or The Bride stripped bare by her bachelors, even. This piece, together with its accompanying Green Box of notes (Duchamp, 1934) and later work Étant Donnes, is generally regarded to be both the culmination and the summation of his work, occupying his thoughts between 1912-1923, when he abandoned it as finally unfinished, leaving us with a seemingly unfathomable puzzle. Both of my early works, Deconstructing Duchamp and StarGlass, transcoded the Large Glass into hypermedial art systems. Deconstructing Duchamp transposed the Large Glass across the internet with 25 collaborators through 25 websites and then interrelated Duchampian images with his Green Box of notes etc., into discreet offline systems such as the StarGlass piece (Harrison, 1997, 2000). As with the original Large Glass, StarGlass is concerned with interpretation through the formulation of 'meaning' derived from the connection of thoughts and ideas into whole concepts. These pieces were made using hypermedia as this enables the linking of multimedia items by semantic association rather than by indexing or alphabetic ordering. Connectivity by semantic association is paralleled in Conceptual Art where ideas are semantically linked by the artist into one concept - often presented as a visual statement, an object. Hypermedia allows for the linkage of interrelated, multi-media ideas into a semantic network, as conceptual art work.

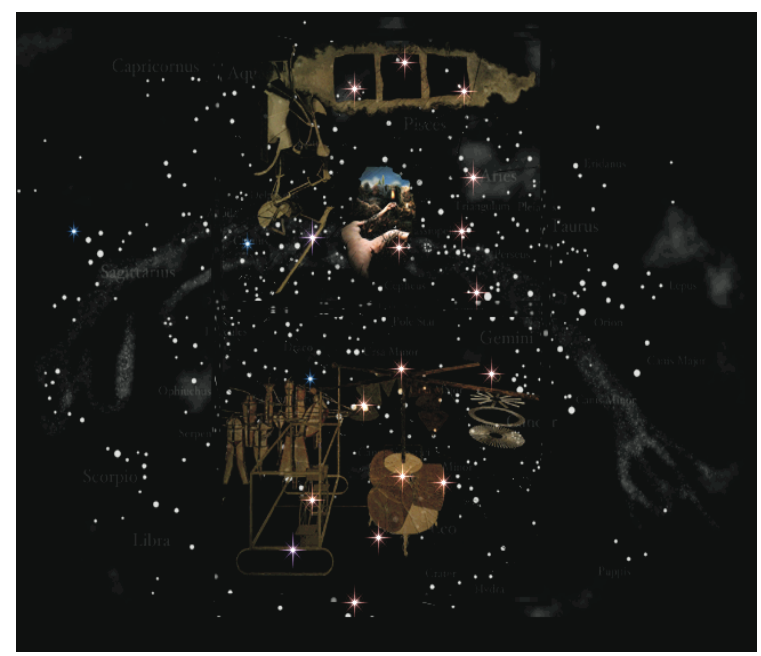

Figure 1: Screen grab from StarGlass - Dew Harrison

\subsection{The Large Glass}

The Large Glass together with the boxes completes a corpus of non-linear, semantically associated ideas ripe for transposing into hypermedia. The Large Glass is the encasement of a plethora of non-sequentially inter-connected ideas and the transposition of these into a new media enables new readings of his work. When seen as a whole entity his work is riddled with cross-references and complex meanings generating different interpretations through its blatant ambiguity. The Large Glass and its semantic key, the Green Box, of 93 documents, sketches, calculations and notes together contain a wealth of association links proffering the conjunction of images and text ideal for hypermedia. The white box, a l'infinitif, (Duchamp, 1966) mostly refers to his thoughts on the fourth dimension. Duchamp's work can be taken as a richly endowed semantic network, which continues to inform contemporary Conceptual artists.

The Large Glass was originally constructed in the form we know early last century; this glass encasement of connected ideas was the nearest Duchamp could get to his goal. The technology was not sophisticated enough at that time to support his interest in the $4^{\text {th }}$ dimension. He wanted to portray his Bride in the $4^{\text {th }}$ dimension and began with painterly abstractions of the figure culminating 
in the flatness of glass as a material nearing the state of no thickness or 'inframince' and therefore acting as a signifier to the $4^{\text {th }}$ dimension. He replaced traditional (thick) paint and canvas as tools for picture making and renounced painting, declaring his Large Glass to be 'a threedimensional physical medium in a fourth dimensional perspective' (Duchamp, 1966).

From Duchamp's notes it would seem that his interest in the $4^{\text {th }}$ dimension was not aligned to the, then contemporary, 'relativity theory' proposed by Einstein but to the idea that the $4^{\text {th }}$ dimension could be understood through geometry progressing from the $\mathrm{n}$-dimension and aligned to the mathematics of Poincaré.

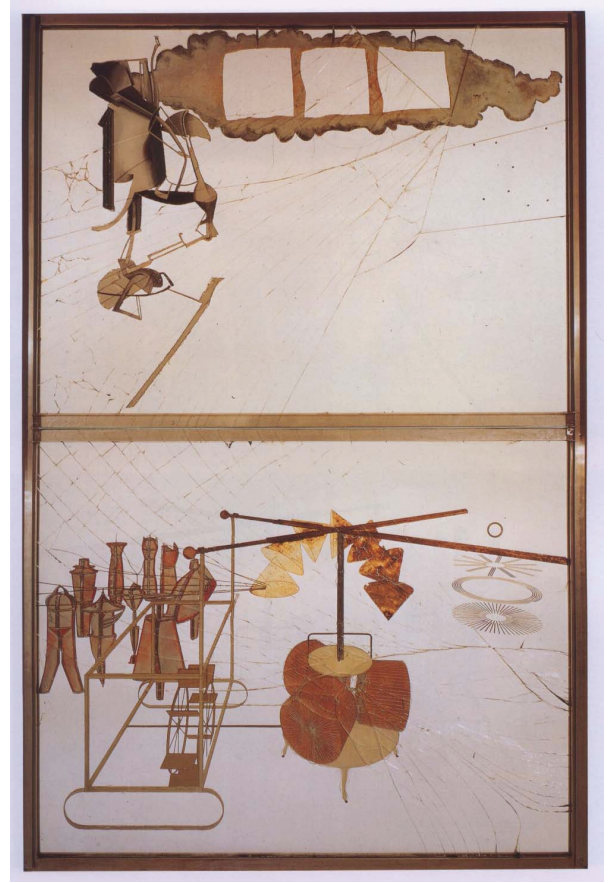

Figure 2: La Mariée mise à nu par ses célibataires, même (The Large Glass) - Marcel Duchamp, 1915-23

A single point has no (n) dimensions, two points define a line and have one dimension, two lines create a plane and have two dimensions, two planes create a volume or a three dimensional space or object, so what do two volumes create? Duchamp suggested that they should create a fourth dimensional space or object. Western art has been traditionally concerned with 2D representations of 3D spaces. Duchamp considered that if $2 \mathrm{D}$ images could stand for a world of 3D objects it would follow that 3D objects could represent things in a 4D world. He conceived the Bride as a 3D representation of a 4D being, as a 'two-dimensional representation of a threedimensional bride who herself would be the projection of a four-dimensional bride in the threedimensional world.' (Duchamp, 1966).
Painters are 2D artists working on a flat plane, sculptors are 3D artists working with material objects in real space and now, in the $21^{\text {st }}$ century, we have digital artists working in the $4 \mathrm{D}$ of cyberspace concerned with the virtual space/object incorporating time. My own work brings the Bride in the Large Glass into cyberspace.

\subsubsection{Flocking behaviours}

Early explorations within my own practice have employed digital technology with hypermedial software such as HTML and Director, for the ability to connect multimedia versions of Duchampian items through semantic association. They have encouraged interactivity by mouse click and rollover, the usual internet methods of access and have therefore been hard-linked. Hypermedia technology required mouse and keypad access which brought an unintended game-like element to the pieces. More recent works have brought in gestural and intuitive action to engage with these ideas, and I am currently experimenting with notions of constant flow and flux in place of set links. In particular I am working toward bestowing the Duchampian items with flocking behaviours in order to interrelate them semantically, the result being more akin to a projected animated text/painting in that they are not all interactive, but may require contemplation in considering their shifting positions.

'Flocking' is more usually associated with the collective animal behaviours exhibited by many living beings such as birds, fish, bacteria and insects, but can be more largely understood as the motion of a large number of self-propelled entities. It is considered an emergent behaviour arising from simple rules that are followed by individuals and does not involve any central coordination. The first Artificial Life programme to simulate 'Flocking;' birds was developed by Craig Reynolds in 1986, his initial paper (Reynolds, 1987, pp. 25-34) he described his Boids simulation as adhering to a set of simple rules:

Separation: steer to avoid crowding local flockmates (short range repulsion)

Alignment: steer towards the average heading of local flockmates

Cohesion: steer to move toward the average position of local flockmates (long range attraction)

More complex rules could then be added, such as obstacle avoidance and goal seeking. Central to the model is the observation that flocks form without a leader but the fluid, lifelike behaviour of the 'Boids' or birds, is produced entirely by deterministic rules. Each flock is dynamic and once together, is not guaranteed to keep all of its members. After running the model for a while, all of the birds have approximately the same heading 
and sometimes a bird breaks away from its flock. Unexpected behaviours, such as splitting flocks and reuniting after avoiding obstacles, can be considered emergent.

Flocking is now widely used in animation and screensaver design and can be achieved through software as regular as Flash. It is commonly seen in films such as Tim Burton's Batman Returns (1992) featuring flocking penguins and Disney's The Lion King (1994) which had a wildebeest stampede. Flocking behaviours can be applied to animal-like and non-animal-like entities and the first experiments with Duchampian items are simple rather than complex, using 'Flash' to animate them. The next phase of Flocking brings a new challenge involving an $\mathrm{Al}$ programmer who is providing more complex behaviours in order to show the criticality of oscillation required in determining the semantic families and shifting relationships within the Duchampian universe of objects. These are text only objects and provide a meta-language of semantic understanding concerning Duchampian ideas, with the object/behaviour patterns lying underneath and of less interest to the viewer. They illustrate the flux and flow of ideas and as such could enable any discourse brought into this system.

\subsubsection{Flocking Duchampian objects}

The moving image projections further displayed the relationships between the thoughts and ideas of Duchamp in a more natural and intuitive way than 'point and click' or 'roll-over' interaction. It is these works which have led to the hands-on installation, 'Shift-Life' in that they employed Al programming to animate their content. The animated non-linear films incorporated the 'swarming' and 'clustering' of the content of his boxes of notes with seminal images from his body of work. By applying Al 'behaviours' to these Duchampian objects they moved towards or away from each other according to their semantic relationships when in close proximity. An over-simplified example is: the word 'rain' may move away from 'stone', until the word 'hail' appears and oscillates between 'stone' and 'rain'. As the multimedia items required for these experiments had been re-cycled from the earlier hypermedia projects, they were really ready-mades newly re-connected into a more organic and dynamic display of the relationships between them. The new 'flocked' Duchampian objects more clearly show the 'families' of images, texts etc., and the oscillations that occur between them due to the pull of their similar semantic positions. Interactivity is incorporated to enable viewers to move the items around and drop-in others, in order to find out how they re-position within the Duchampian mindset.

Experiments where 'flocked' objects as text only items, now cluster words and sentences into positions to further make sense of Duchampian non sense. Text and language are the tools of a postDuchampian conceptual practice. New text strings can apply to any current art debate where words can be added by the viewer to sway an argument, keeping it alive and dynamic. The overall argument pattern should evolve from the individual behaviours of the text objects.

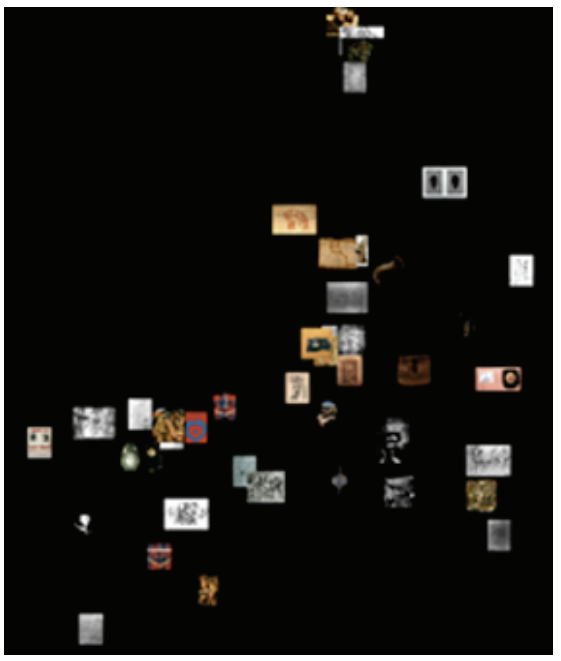

Figure 3: 'flocking' Duchampian objects

\section{DARWIN}

This recent work on Duchamp has directly influenced the approach to the exploration of Darwin's 'big idea', in that instead of Duchampian objects, Al behaviours were attached to a virtual world of animated objects featured as creatures and plants that adapt to Darwinian principles for survival. These 'families' were grouped by their form and colour, and it is the changes in these identifiers that are of interest when observed by viewers who can cause physical upheavals in their world, to which the artificial families of life-forms then have to respond. The first phase of the ShiftLife project entailed a fantasy biological life-form, 'bugs-in-a-box', existing in conditions analogous to Darwinian evolution. Interfering with the stability of their ecosystem was done through real world actions directly affecting the virtual one. The work relied heavily on the 'animal intelligences' programmed in to display accelerated Darwinian principles and the piece was made in collaboration with Dr Eugene Ch'ng an artificial life programmer, Sarah Mount a sensor developer and Samantha Moore an artist known for her 2D animated films.

2009 was the $200^{\text {th }}$ anniversary of the birth of Charles Darwin in Shrewsbury and the $150^{\text {th }}$ anniversary of the publication of On the Origin of Species. As part of the national celebrations underway, the interactive installation Shift-Life was 
commissioned and exhibited at Shift-Time - a festival of ideas in Shrewsbury, summer 2009. The work was then shown at the Wolverhampton Art Gallery January-March 2010. It is still in its prototype beta-testing stage and, following this, will be modified and enriched with extra behaviours and more sensitive environmental changes as we develop the project to more closely demonstrate Darwinian ideas for further exhibition.

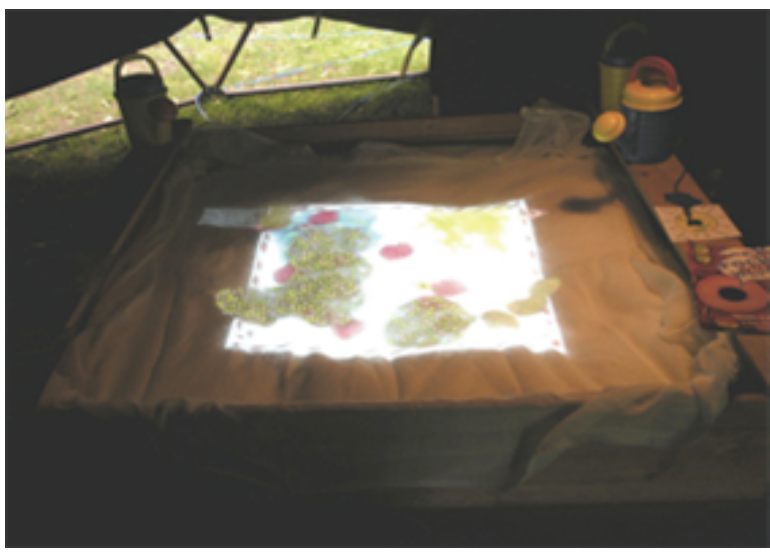

Figure 4: The Shift-Life installation at Shrewsbury

In response to Darwin's idea, the aim of this work was to create an 'alternate' biological life as a set of artificial or virtual organisms that possess similar biological processes to their 'real' counterparts, such as growth, reproduction, and adaptation. The virtual life forms exist in a nutritional (trophic) relationship of prey/predator, and include both rooted (sessile) and free ranging (vagile) organisms. Animal-intelligence was programmed into the virtual organisms to allow them survival strategies. The project also involved the construction of an enhanced mixed reality-based virtual environment to support the organisms. The climate of the virtual environment was directly influenced by the data gathered by wireless sensors (gumstix) in the real world landscape (bead box), plus implements (lights, shakers, pourers...) that altered the parameters (temperature, humidity, acidity, stability...) and so allowed visitors to change the condition of the virtual landscape.

The installation comprised of a large wooden 'sandpit' box filled with polystyrene beads representing the virtual world terrain, this encouraged interactivity for visitors who could physically manipulate a set of implements to radically alter the living conditions of the fantasy creatures in their virtual ecosystem, projected into the installed box space. By pouring liquids, switching on lights, moving objects etc., into and around the box, visitors could see immediate responses to their actions played out in the animated ecosystem as the projected bug-like life forms adapted to survive.
Interacting with the real world landscape and observing the instant affect a visitor's actions had on the creatures, proffered an understanding of how causing changes in environmental conditions, forces evolutionary developments on the resident life-forms.

In attempting to both respond to the idea of a young Darwin and to elucidate his thinking in an holistic hands-on way, the bug-like creatures in the box were reminiscent of his/any childhood and so took the form of jelly sweets and allsorts. Darwin was born and spent his childhood in Shrewsbury, where he began his observation of natural lifeforms and started his vast collection of beetles. This boyish interest would last all his life and led to his great insights later on as an adult. The virtual world of 'bugs-in-a-box' aesthetic was developed by the Shift-Life animator.

Moore created the 'sweet' creatures with a view to making them as approachable as possible to an audience diverse in gaming/Al experience. The images moved away from a computer-games visual aesthetic of hyper-reality and towards a deliberately non-digital, non-microbe aesthetic. The creatures are flat, bright, and cartoon-like cute. This positioned her work in the realms of the overtly rather than covertly 'made', and referenced a clear fantasy world instead of attempting photo realism. The bugs were based on pick and mix sweets; the carnivore was a liquorice all-sort, the herbivore was a jelly sweet and the foliage (for shade, sustenance etc.) was based around a selection of penny sweets.

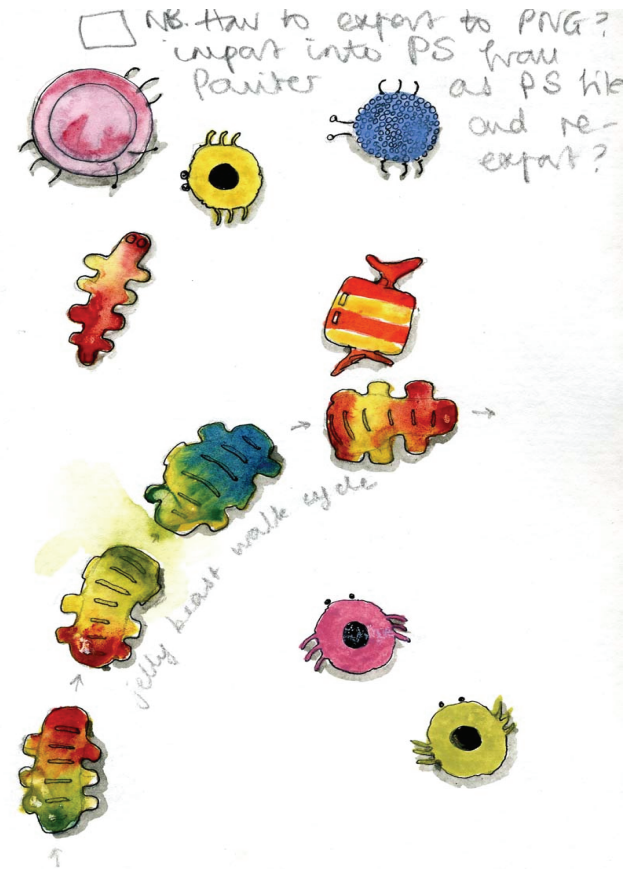

Figure 5: Preliminary sketch of sweet bugs 
The creatures were limited to two dimensions as they were to be observed from above, and in collaboration with the bio-life programmer a set of characteristics, limitations and behavioural patterns were decided upon and integrated into the design. For example, the herbivores were much larger than their predators and had better eyesight. The carnivores though, despite being small and short sighted, had a fast scuttling gait compared to the lumbering movement of their prey so the relationship between them was not as uneven as it may have first appeared. Moore found that animating to the agreed behavioural perimeters was interesting and frustrating in equal measure; some behaviours (such as procreation or multiplication of the creatures and fighting or cannibalism between carnivores) were able to be animated, but were not included into the software programme as the final animation was too complex to be smoothly integrated. Moore and Ch'ng hope to resolve these issues in the next stage of the project.

Ch'ng's recent programming work on enhanced Virtual Environments (eVE) and in particular climate-change and biodiversity research for terrestrial and marine environments identified him as the prime candidate for contributing animal behaviour and adaptability to the Shift-Life work. The system was constructed with a trophic network of predator-prey and vegetation. The organisms were short-lived (60 seconds minimum and 150 seconds maximum). The general behaviour of each organism concerned its survivability and the reproduction of progenies. The survival of the entire ecosystem depended on the balance of the organisms that inhabited the landscape. If the predator out-grew the prey, an imbalance would occur and the system would perish. If the prey outgrew the edible plants, the food would be scarce and the system in a dilemma. If the canopies (large trees) over-reproduced, the predator had little space left to hunt. If the red poison plants outgrew the vegetation, more herbivores would eat them and so become toxic; consequently their newly acquired toxicity would kill the carnivores (see Figure 6). When the environment particularly suited them, a plant species would thrive (the poison plants loved high $\mathrm{pH}$ levels).

The difficulty of such an alife development was the maintenance of equilibrium. The 'fun' part was when the users interacted with the system by increasing any of the environmental factors via our sensor network: temperature, sunlight, humidity, $\mathrm{pH}$ level. They could do this by using watering cans, hammers, and lamps. The fitness of each organism was measured by Ch'ng's Adaptability Measure (Ch'ng, 2007). The creatures were affected by three environmental factors: temperature, sunlight and humidity and the plants were affected by six factors: temperature, sunlight, soil, humidity, $\mathrm{pH}$ level, and space. The temperature, sunlight, humidity and $\mathrm{pH}$ level were streamed from electronic sensors installed at strategic locations in the virtual bug box. Soil conditions were unchanged and were based on height fields (certain regions are more habitable). The availability of space for plant growth depended on how many of them shared the space.

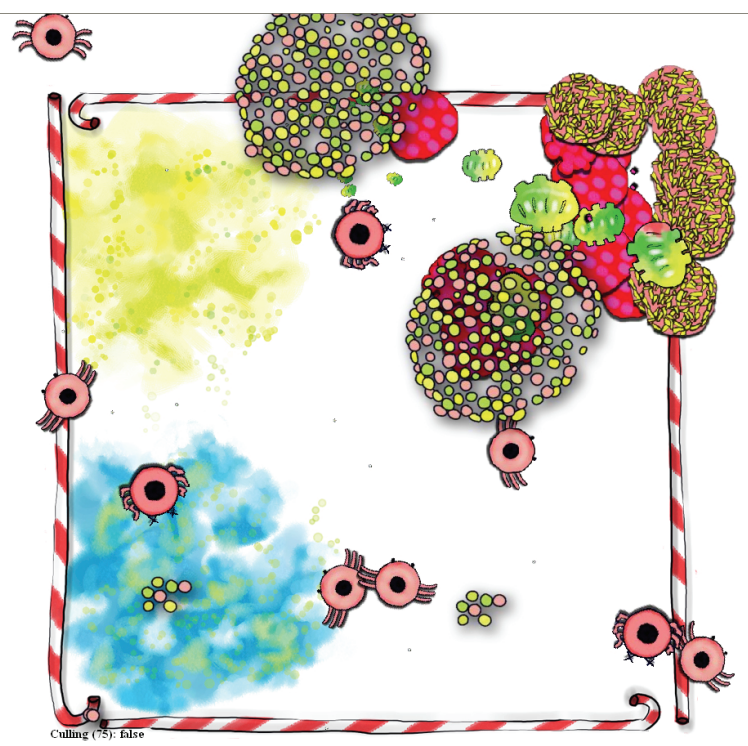

Figure 6: Shift-Life virtual world life-forms

In order to interact with the virtual 'sweet' bugs they were projected down into a real box arrayed with sensors. This was a wooden 1.2 metre sq. box filled with polystyrene beads held under a muslin sheet and surrounded by a set of manipulative tools which, when activated, could affect in realtime the projected virtual world of creatures causing them to adapt to survive in their rapidly changing ecosystem. The tools were for hammering earthquakes into existence, watering to alter the humidity and $\mathrm{pH}$ count of the planet environment and altering light sources to affect temperature and depth of shade.

The physical 'bug box' was a pervasive computing application with an interface consisting of the following apparatus:

- An Oak USB attached 3-axis accelerometer hidden underneath an icon attached to the side of the bug box and a toy hammer with which to actuate the sensor. The output of the sensor was normalised and an exponential weighted moving average applied to smooth the response from the transducer;

- An Oak USB luminosity sensor, actuated by 
an angle-poise lamp;

- An Oak USB relative humidity sensor, protected by a Gortex membrane, actuated by pouring water into the bug box, and

- A Phidgets USB pH sensor, actuated by pouring vinegar and bicarbonate solutions into the box.

The sensor network was centralised and reported back in a best-effort fashion. A number of optimisations were made to enable the application to appear to be responding immediately. In particular, unnecessary reports were suppressed, and each datum only reported back to the ecosystem if it differed from the previous reading by a threshold.

In order to meet the commission and elicit an understanding of Darwinian adaptation through interactive installation an intuitive interface was required with the potential for tangible physical activity. The wooden virtual bug box was large enough to allow for small groups of people, families, and individuals to interact with it and with each other, it was accessible to both children and adults. The box was equipped with sensors to respond to the visitors' actions and relate their physical activities directly to the virtual ecosystem projected into it. As they poured water, for instance, the humidity would alter and some plants may die back, this would mean less food for the herbivore green jelly sweet bugs, and consequently less bugs to eat for the pink carnivores. Switching the lamp on would dry out the atmosphere and enable plants to grow again. However, too much 'sun' might be detrimental to the point of wiping out the carnivores entirely. They could, in fact, become extinct due to their reproduction method of cloning, unlike the egg laying herbivores. When this occurred, we had to re-start the programme to reassure the smaller children that they weren't responsible for a complete genocide! Pouring vinegar (poison) from a watering can would 'feed' the red bushes, toxic to all the creatures, but this could be remedied by pouring baking soda liquid (plant food) and restoring the plant balance, the herbivores' main food source. Hammering on the box sent the carnivores into panic mode and they would spin around and run for cover under the trees.

We ran the installation for two days in a dome sited outside in the Quarry Park for the Shrewsbury event and two months inside at the Wolverhampton Art Gallery. Our observations and interviews showed that all our visitors, young and old actively contributed as participators by physically moving the box objects to change the parameters that affected the projected virtual world. Perhaps more importantly, they also often remained for some time in a state of reflection to passively observe the other's actions and watch the life-form changes taking place. The jelly bug world was set at a selfsustainable and stable level without the intervention from human meddling and as such was visually mesmerising, it was essentially a sugar-coated version of 'nature red in tooth and claw'. There was room for contemplation where the virtual world could be understood as an analogy for human activity and its effect on global climate change within our own real world. This became evident through the participants' conversations. The tangible 'hands on' interface proved to be instinctive, attractive and informative on many levels, delivering a good example of an Arts-Sci collaboration, but in this first stage, there were many questions about the behaviours and environmental conditions which we hope to reduce in the next phase.

Exhibiting Shift-Life in the clean gallery setting at Wolverhampton was more problematic. The constant flow of visitors activating the sensors by hammering, pouring liquids etc., for a longer showing with more participants, needed more robust methods of protecting the sensors, a set of spares and a more efficient way of dealing with the excess liquids collected underneath the display. A pump was connected to clear the waste liquids collected from the watering cans. Phase 2 of the project needs to address the installation as a possible 'closed' or self-sustaining water cycle system. The team intends to programme/animate the creatures not only to rapidly evolve and change physically in accordance with their volatile world, but to evolve unpredictable social patterns from their individually programmed behaviours. The project is also to extend to include small 'sensor' creatures which will crawl around with the virtual bugs.

\section{REFERENCES}

Ch'ng, E. (2007) Modelling the Adaptability of Biological Systems. The Open Cybernetics and Systemics Journal, 1. pp. 13-20.

Duchamp M. (1934) The Green Box. Edition Rose Sélavy, Paris.

Duchamp. M. (1966) A l'Infinitif. Cordier \& Ekstrom, New York.

Harrison, D. (1999) Mind, Memory, Mapping, Metaphor: Is Hypermedia Cognitive Art? In: Ascott, R. Ed. Reframing Consciousness: Art, Mind \& Technology, Intellect Books, Exeter UK. pp. 151155. Reprinted Sept 2000.

Harrison, D. (1997) Hypermedia as Art System. In Drucker, J. (ed.) Digital Reflections: The Dialogue of Art and Technology. Art Journal, Fall, 56 (3). pp. 55-59 
Reynolds, C. (1987) Flocks, Herds, and Schools: A Distributed Behavioral Model. In Computer

Graphics, 21(4) SIGGRAPH '87 Conference
Proceedings. pp. $25-34$

Tamblyn, C. (1990) Computer Art as Conceptual Art. Art Journal, Fall, pp. $253 \mathrm{ff}$. 